\title{
AVALIAÇÃO DE FERRAMENTAS WEB DISPONÍVEIS PARA O GERENCIAMENTO DE PROJETOS A PARTIR DE JULGAMENTOS ONLINE DE USUÁRIOS
}

\author{
Camila Corrêa de Melo (UFPE) camilacorreamelo@gmail.com
}

\section{Resumo}

Este trabalho analisou cinco softwares de Gestão de Projetos mais bem avaliado no mercado atualmente e buscou estimar a usabilidade deles a partir de avaliações online de usuários que se utilizavam dessas plataformas para o planejamento e controle dos seus projetos. Foram identificados os recursos recorrentemente citados nas avaliações, considerados como importantes à luz dos usuários, e os classificou em cada área de conhecimento de gerenciamento de projetos. Dessa maneira, foi possível reconhecer as lacunas existentes entre as ferramentas utilizadas para o gerenciamento de projetos e propor melhorias que garantissem o controle mais eficiente dos projetos em uma organização.

Palavras-Chaves: Sistemas de Informação, Software, Projetos, Tecnologia, Sistemas colaborativos

\section{Introdução}

Uma grande quantidade de informações em projetos precisa ser gerenciada em razão do seu escopo, complexidade e o número elevado de pessoas envolvidas, isso faz com que as empresas recorram a softwares que as ajudem na gestão da informação dos seus projetos (SOFTWARE ADVICE, 2019).

No entanto, de acordo com a pesquisa realizada com mais de 300 organizações pela Wellingtone Project Management (2018), 55\% das organizações não têm acesso em tempo real dos indicadores do projeto e normalmente $50 \%$ delas gastam de um ou mais dias para coletar todos os dados do projeto. Também, foi identificado que as ferramentas popularmente utilizadas para o gerenciamento dos recursos de um projeto são Planilhas de Excel, seguido por $22 \%$ que utilizam um software de gestão de projetos (WELLINGTONE, 2018).

Os benefícios pelo uso de ferramentas de gerenciamento de projetos são largamente abordados. Segundo o relatório da Pulse of the Profession (2019) a integração da tecnologia de informação de projetos e dos profissionais com habilidades certas, produz oportunidades para o controle dos projetos, além de produzirá benefícios para o controle dos projetos, além 
de empreendimentos únicos melhor alinhados a estratégia organizacional e com pessoas engajadas.

Uma pesquisa realizada pela Capterra (2019), na qual 200 usuários de software de gerenciamento de projetos foram entrevistados, vários benefícios pelo uso de softwares de gerenciamento de projetos foram levantados, dentre eles destacam a conclusão do projeto no tempo planejado e cumprindo o orçamento, qualidade do produto final, colaboração do time, visibilidade das atividades realizadas, acuracidade nas estimativas de tempo e recursos e capacidade de gerenciar as expectativas dos clientes.

Assim, se constatou que softwares de gerenciamento de projetos podem auxiliar os gerentes no controle e monitoramento dos projetos quanto aos pilares fundamentais de tempo, custo e qualidade (FinancesOnline, 2019), sendo ferramentas que auxiliam na gestão de tarefas, recursos (humanos e financeiros), mudanças ao longo do ciclo de vida do projeto, entre outras atividades fundamentais em projetos.

Apesar da literatura ressaltar a importância da gestão da informação como um fator que influencia o sucesso do processo de um projeto (LUPAŞC, 2017; DUBININA E HRYTSIAK, 2018; TAHERKHANI, 2018), a discussão focada nas ferramentas que apoiam as atividades de planejamento, monitoramento e controle não é um assunto recorrente entre os trabalhos.

Estudos relacionados a aplicação de softwares comercialmente conhecidos da área de projetos foram facilmente encontrados, dentre eles o MS Project, colaborando para estudos de riscos (BUDAYAN ET. AL, 2018); e Primavera, apoiando o desenvolvimento de sistema para o gerenciamento de projetos de construção (GRAKHOV, 2017) e a otimização da alocação de pessoas entre dois projetos de construção (REDDY, NAGARAJU, E SALMAN, 2015). Além disso, observou-se que a maioria destes se baseiam em apenas uma funcionalidade para análise.

Por outro lado, poucos trabalhos envolvendo a comparação de um ou mais softwares foram encontrados, destacamos Wit e Herroelen (1990), Rushinek e Rushinek (1991) e Ilyas, Hassan e Ilyas (2013); também, uma pesquisa recente comparou as ferramentas MS Project e Primavera (KHALIL E SHAMAL, 2019); e um trabalho destacou alguns critérios para a escolha de ferramenta (KACZOROWSKA, SŁONIEC E MOTYKA, 2017).

Em razão da grande variedade de ferramentas para o gerenciamento de projetos disponíveis no mercado e as poucas abordagens de comparativos entre softwares de gerenciamento de 
projetos, este trabalho sugere revisar as funcionalidades presentes entre as ferramentas mais populares do mercado e compará-las, valendo-se para isso de avaliações online dos usuários.

Outra motivação para realização deste trabalho é considerar o sistema de informação como uma ferramenta comum a todas as áreas de conhecimento em projetos. Isso revela a importância de avaliar as ferramentas à disposição para o planejamento e controle dos projetos, além de usar dessas informações para a tomada de decisões.

Dessa forma, esse estudo se releva importante para a área de projetos, haja vista que proporcionará a identificar algumas lacunas existentes nesses recursos e levantar oportunidades de melhorias, aprimorando a eficiência do monitoramento e controle dos projetos como também servindo de informações para a tomada de decisões.

Este trabalho está dividido em quatro partes. Nesta primeira parte está descrita uma introdução sobre a temática de softwares de gestão de projetos. A segunda parte traz conceitos relacionados ao sistema de informações em projetos. Em seguida, na terceira seção, expõe a metodologia seguida pelo trabalho e como foi realizada a coleta dos dados considerados para o estudo. Na quarta seção estão apresentadas as análises e discussões realizadas. E por fim, apresenta-se as considerações finais sobre o estudo.

\section{Referencial Bibliográfico}

A seguir serão apresentados os principais conceitos relacionados a temática deste trabalho.

\subsection{Sistema de informação de Gerenciamento de Projetos - SIGP}

Em todos os setores organizacionais, os gerentes empregam tecnologia da informação como um recurso para competir estrategicamente (TURBAN, RAINER E POTTER, 2005). Um sistema de informação extrai, processa, armazena, explora e distribui as informações para atender um objetivo especifico (TURBAN, RAINER E POTTER, 2005), colaborando com as atividades de tomada de decisão pela organização (TURBAN, DELEN E SHARDA, 2014).

Além de suportar a comunicação e colaboração entre a equipe de um projeto, um sistema de informação do gerenciamento de projetos também contribui para monitorar as atividades, controlar as mudanças, analisar e prever o desempenho do projeto, permitir que as partes envolvidas acompanhem o status do projeto, disponibiliza informações no tempo real durante 
todo o ciclo de vida do projeto e facilitar os feedbacks entre os membros do projeto (SY, 2009).

Segundo o Guia PMBOK (2004, p. 377), "Um sistema de informações que consiste de ferramentas e técnicas usadas para reunir, integrar e disseminar as saídas dos processos de gerenciamento de projetos", além de "dar suporte a todos os aspectos do projeto, da iniciação ao encerramento, e pode incluir sistemas manuais e automatizados" (PMBOK, 2004, p. 377).

\subsection{Software de Gerenciamento de Projetos}

Em todos os setores organizacionais, os gerentes empregam tecnologia da informação como um recurso para competir estrategicamente (TURBAN, RAINER E POTTER, 2005). Um sistema de informação extrai, processa, armazena, explora e distribui as informações para atender um objetivo especifico (TURBAN, RAINER E POTTER, 2005), colaborando com as atividades de tomada de decisão pela organização (TURBAN, DELEN E SHARDA, 2014).

Além de suportar a comunicação e colaboração entre a equipe de um projeto, um sistema de informação do gerenciamento de projetos também contribui para monitorar as atividades, controlar as mudanças, analisar e prever o desempenho do projeto, permitir que as partes envolvidas acompanhem o status do projeto, disponibiliza informações no tempo real durante todo o ciclo de vida do projeto e facilitar os feedbacks entre os membros do projeto (SY, 2009).

Segundo o Guia PMBOK (2004, p. 377), "Um sistema de informações que consiste de ferramentas e técnicas usadas para reunir, integrar e disseminar as saídas dos processos de gerenciamento de projetos", além de "dar suporte a todos os aspectos do projeto, da iniciação ao encerramento, e pode incluir sistemas manuais e automatizados" (PMBOK, 2004, p. 377).

\subsection{Software Colaborativo - Groupware}

Os sistemas colaborativos, também conhecidos com C-orientado, considera a comunicação, coordenação e cooperação sob uma estrutura triangular (BORGHOFF E SCHLICHTER, 2000) de acordo com a figura 1 abaixo. Os sistemas colaborativos ajudam na designação de responsabilidades para a equipe e salva o processo de tomada de decisão (BORGHOFF E SCHLICHTER, 2000). 
A coordenação busca encontrar a melhor forma para organizar as atividades e alocar os recursos; a comunicação ressalta a troca de informações entre as pessoas de forma compreensível; e a cooperação, mais demandando entre os três e foca no trabalho cooperativo em projetos e tarefas comuns (ZHENG E POSSEL-DÖLKEN, 2002).

\section{Figura 1 - 3 C Sistemas de Colaboração}

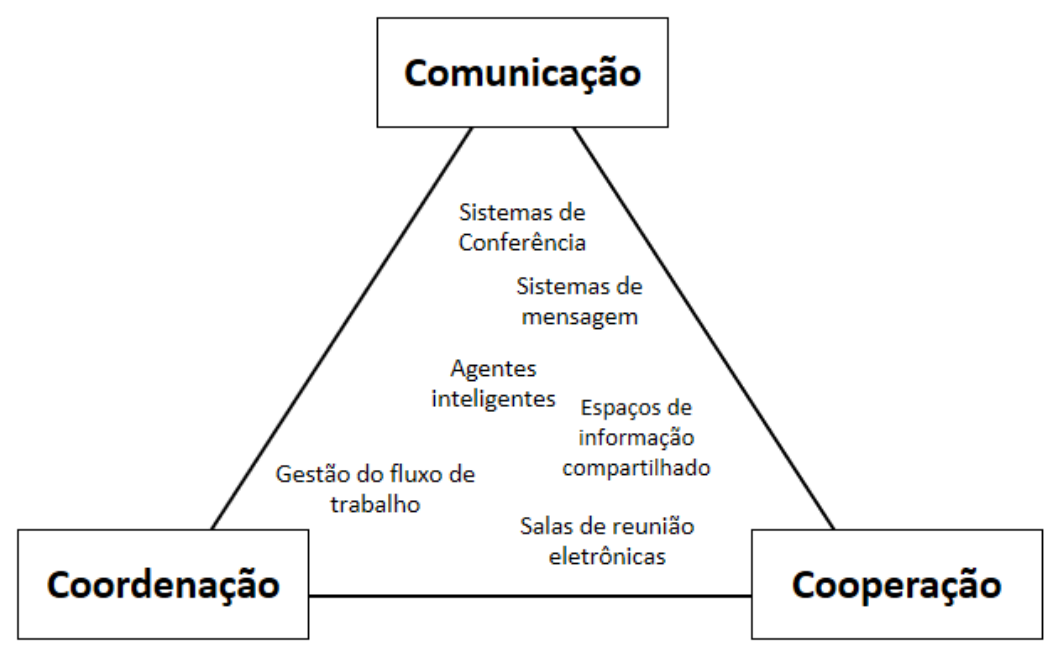

Fonte: Teufel (1995) apud Zheng e Possel-Dölken (2002)

Segundo o Guia PMBOK (2004, p. 377), "Um sistema de informações que consiste de ferramentas e técnicas usadas para reunir, integrar e disseminar as saídas dos processos de gerenciamento de projetos", além de "dar suporte a todos os aspectos do projeto, da iniciação ao encerramento, e pode incluir sistemas manuais e automatizados" (PMBOK, 2004, p. 377).

\section{Metodologia}

Este estudo pode ser classificado quanto à sua abordagem como qualitativo, uma vez que se preocupa em compreender a respeito da usabilidade de diferentes softwares em gestão de projeto por meio de julgamentos pessoais de profissionais que lidam com projetos ou atividades relacionadas no seu dia-a-dia. Em relação a finalidade, o trabalho é uma pesquisa aplicada, pois procura identificar as lacunas existentes entre os softwares de gerenciamento de projetos mais bem avaliados na área e propor alternativas de melhorias para contribuir com a gestão de informação em projetos. No que se diz respeito aos objetivos, o estudo se destaca 
como descritivo visto que almeja reconhecer as características dos softwares para o gerenciamento de projetos e as relações entre eles.

Quanto aos métodos utilizados para a obtenção dos dados realizou-se uma pesquisa documental, a partir de revisões de avaliações eletrônicas reunidas em plataformas que julgam os softwares, levantando em consideração os diferentes pontos de vista positivos e negativos dos profissionais a respeito dos softwares considerados neste estudo.

\subsection{Coleta de dados}

A coleta de dados seguiu quatro etapas: levantamento, listagem, filtragem e avaliação dos dados relevantes.

$\mathrm{Na}$ primeira etapa desse estudo foi realizado o levantamento dos principais softwares e ferramentas que foram analisados nas etapas subsequentes. Para isso, utilizou-se um buscador online a fim de encontrar diferentes listas com os nomes dos softwares de gerenciamento de projetos mais populares no mercado. Ao final, foram avaliadas as dez primeiras listas encontradas pelo resultado de busca.

Em seguida, catalogou-se em uma única lista todos softwares e ferramentas resultando em um total de 153 plataformas. Posteriormente, avaliou aquelas plataformas mais recorrente e fez-se uma nova relação considerando aquelas que apareceram pelo menos 4 vezes entre as listas. Ao final, considerou-se um conjunto de 15 ferramentas de gerenciamento de projetos.

A partir desse último grupo, fizemos uma última filtragem, na qual cada ferramenta foi avaliada individualmente, segundo às descrições em seus websites, e chegamos a uma lista final de 5 softwares listados na tabela 1 . Nesta etapa, aquelas ferramentas que foram descritas como "gerenciador/gestão de tarefas", "ferramenta de acompanhamento" ou "ferramentas de colaboração" foram desconsideradas, haja vista que o objetivo deste estudo é encontrar ferramentas que vão além da função de gerenciador de tarefas ou recursos humanos, somente; mas que possam apoiar o gerente de projetos na tomada de decisão nas diversas áreas de conhecimento do projeto.

Por exemplo, apesar das plataformas Asana e Trello serem as mais citadas entre as listagens, mas foram desconsideradas do estudo, pois, de acordo com as declarações, enquadra-se à classe de ferramentas de gestão de tarefas e colaboração, respectivamente. 
Tabela 1 - Softwares de Gerenciamento de Projetos

\begin{tabular}{|c|c|}
\hline Software & Descrição \\
\hline Zoho Projects & $\begin{array}{l}\text { Software de gerenciamento de Projetos inclui recursos como } \\
\text { automatização e gerenciamento das tarefas, acompanhamento do } \\
\text { tempo e problemas, gerenciamento de fóruns sociais e a administração } \\
\text { do projeto. }\end{array}$ \\
\hline Wrike & $\begin{array}{l}\text { Software de gestão de Projetos, criado para Gerentes de Projetos, } \\
\text { inclui as ferramentas de gráfico de Gantt, gestão de recursos e carga } \\
\text { de trabalho, status e fluxo de trabalho, painéis visuais e relatórios e } \\
\text { ajuda a determinar as prioridades e alinhar a equipe para as atividades. }\end{array}$ \\
\hline $\begin{array}{l}\text { Teamwork } \\
\text { Projects }\end{array}$ & $\begin{array}{l}\text { Software de gerenciamento de projeto que permite que se tenha uma } \\
\text { visão geral de todo o trabalho, ajuda na entrega dos resultados e torna } \\
\text { o trabalho da equipe do projeto mais fácil. }\end{array}$ \\
\hline LiquidPlanner & $\begin{array}{l}\text { Uma solução de gerenciamento de projetos preditiva a partir de uma } \\
\text { programação dinâmica e gestão dos recursos, disponibilizando uma } \\
\text { estimativa acurada sobre a conclusão do projeto. }\end{array}$ \\
\hline $\begin{array}{l}\text { Freedcamp } \\
\text { Software }\end{array}$ & $\begin{array}{l}\text { Sistema de gerenciamento de projetos que oferece várias ferramentas } \\
\text { que uma equipe precisa para completar seus projetos com sucesso. }\end{array}$ \\
\hline
\end{tabular}

Fonte: Autor (2019)

\section{Análise de dados e discussões}

A seguir serão apresentadas algumas discussões a partir dos dados observados nos websites das plataformas e especializados em avaliações de softwares.

Quanto ao perfil dos usuários das avaliações, verificou-se que aqueles que mais se utilizavam dos recursos dessas plataformas trabalhavam no setor de tecnologia e marketing/comunicação, equivalendo a 53\% das avaliações, enquanto apenas $4 \%$ das avaliações representaram os julgamentos de profissionais das empresas tradicionais como indústria e automotiva e o restante $43 \%$ foi distribuindo entre vários setores como consultoria, finanças, educação, entre outros. O gráfico 1 os dados anteriores. Além disso, constatou que essas plataformas eram majoritariamente utilizadas em organizações pequenas com até 50 pessoas. Em relação ao tempo de uso, muitas organizações relataram que utilizavam essas ferramentas há pelo menos dois anos. 


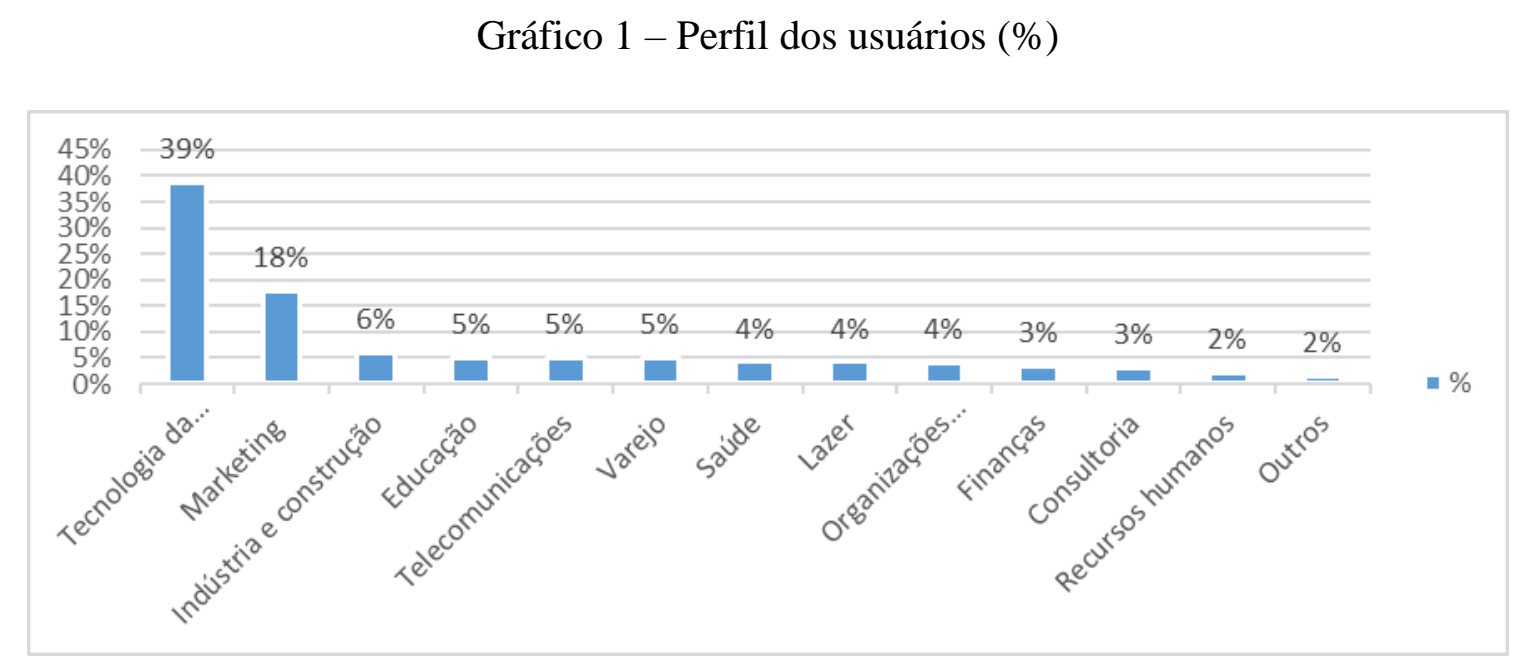

Fonte: Autor (2019)

Os resultados foram baseados na análise de 2264 sentenças acerca das avaliações dos cinco softwares de gestão de projetos considerados neste estudo. Deste total, 1825 sentenças são julgamentos positivos, os quais os usuários relatavam pontos positivos a respeito das funcionalidades das plataformas, por outro lado 439 sentenças revelam considerações negativas, em razão da plataforma não atender a alguma função vista como necessária pelo usuário.

Essas sentenças foram agrupadas em 60 e 68 classes de abordagens positivas e negativas, respectivamente. Ou seja, julgamentos que expressavam de formas diferentes uma mesma funcionalidade foram representados por uma frase ou palavra, por exemplo "interface intuitiva" e "design intuitivo" foram agrupadas sob a funcionalidade de "interface intuitiva".

Algumas funções foram classificadas de acordo com as áreas de conhecimento em gerenciamento de projetos e verificou-se quais delas os usuários mais enfatizaram nas suas avaliações. A tabela 2 a seguir resume esses dados. As áreas de integração e aquisições não foram consideradas na tabela. No que se diz respeito a área de integração, consideramos que a plataforma é a ferramenta que integra todas as áreas do projeto, nos dando uma visão holística do projeto. Enquanto a área de aquisições, não foram constatados registros entre as avaliações.

Tabela 2 - Percentual de funcionalidades de acordo com as áreas de conhecimento

\begin{tabular}{|l|l|l|l|}
\hline \multicolumn{1}{|c|}{$\begin{array}{c}\text { Áreas de } \\
\text { Conhecimento }\end{array}$} & $\%$ & Áreas de Conhecimento & $\%$ \\
\hline Plano de & $6 \%$ & Plano de gerenciamento de & $21 \%$ \\
\hline
\end{tabular}




\begin{tabular}{|c|c|c|c|}
\hline $\begin{array}{l}\text { gerenciamento do } \\
\text { Escopo }\end{array}$ & & Recursos Humanos & \\
\hline $\begin{array}{l}\text { Plano de } \\
\text { gerenciamento de } \\
\text { Tempo }\end{array}$ & $38 \%$ & $\begin{array}{l}\text { Plano de gerenciamento das } \\
\text { Comunicações }\end{array}$ & $19 \%$ \\
\hline $\begin{array}{l}\text { Plano de } \\
\text { gerenciamento de } \\
\text { Custos }\end{array}$ & $1 \%$ & $\begin{array}{l}\text { Plano de gerenciamento de } \\
\text { Riscos }\end{array}$ & $1 \%$ \\
\hline $\begin{array}{l}\text { Plano de } \\
\text { gerenciamento de } \\
\text { Qualidade }\end{array}$ & $15 \%$ & & \\
\hline
\end{tabular}

Observou-se pelos comentários dos usuários que recursos para o planejamento, monitoramento e controle do tempo (42\%), seguido de recursos humanos (19\%) e comunicação (19\%) prevaleceram entre as funcionalidades das plataformas de gerenciamento de projetos. Por outro lado, as funções para o gerenciamento de custos $(1 \%)$ e riscos $(1 \%)$ foram as menos abordadas nos discursos positivos.

Dentre as funcionalidades mais consideradas entre as avaliações em relação as áreas de conhecimento, destacamos primeiramente a facilidade dos usuários para utilizar a plataforma, em razão da sua interface amigável e recursos intuitivos, por exemplo, seguido; em segundo lugar, pelos recursos gráficos para acompanhar a progressão em tempo real do projeto e suas atividades; e, em terceiro, as funções de favoreceram para um ambiente colaborativo, permitindo que toda a equipe tenha uma visão geral de todas as informações do projeto.

Outras funcionalidades dissociadas às áreas de conhecimento também foram consideradas em várias avaliações, dentre elas destacamos a interação da plataforma com outros softwares, as opções para personalizar as funções disponíveis de acordo com as necessidades dos usuários, a função calendário que faz a integração entre diferentes áreas de conhecimento de projetos e o recurso de privacidade. O gráfico 2 abaixo mostra a representatividade dessas funções nas avaliações dos usuários. 
Gráfico 2 - Representatividade em \% de outras funcionalidades

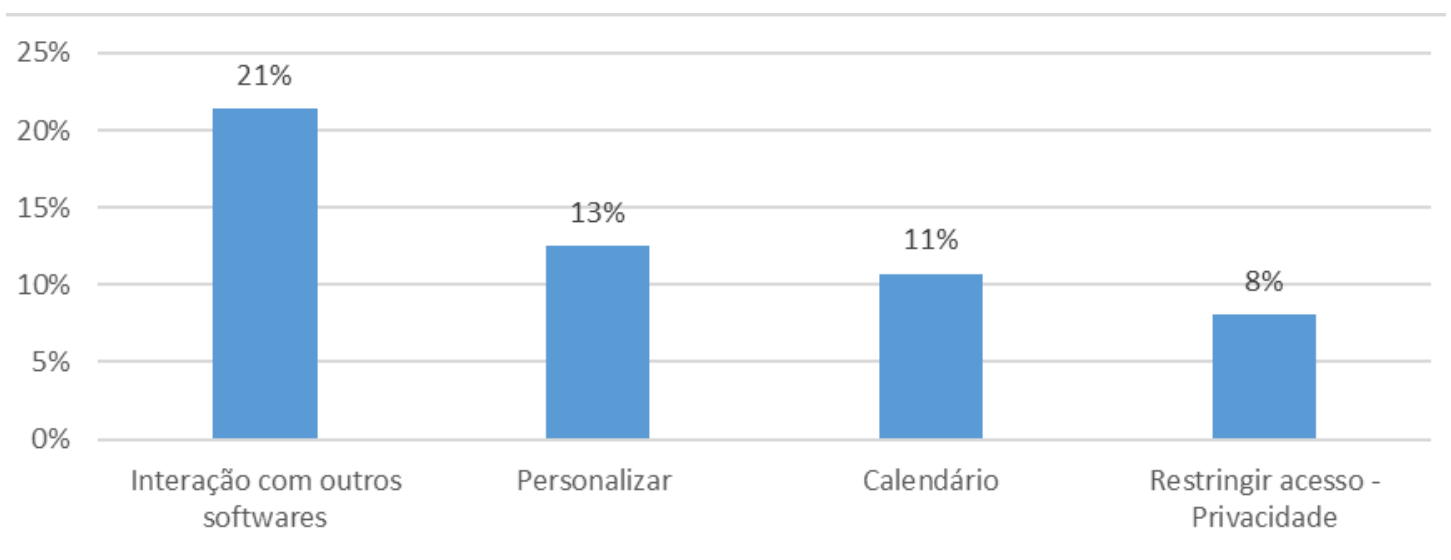

Fonte: Autor (2019)

No que se refere aos pontos negativos, inicialmente foram agrupados de acordo com cada área de conhecimento (ver gráfico 3). Observamos que a insatisfação dos usuários quanto as funções disponíveis nas plataformas de gerenciamento de projeto se concentram nas três principais áreas consideradas anteriormente, recursos humanos, comunicação e tempo, respectivamente.

A alta curva de aprendizado das plataformas é considerando um problema por muitos usuários em suas avaliações, impactando diretamente a familiaridade e aceitação da plataforma pelas pessoas envolvidas no projeto. A ausência de recurso que facilitasse e tornasse a comunicação mais rápida - chats online, por exemplo - como também funções para o melhor controle do tempo - contador de horas (timer log), por exemplo - foram atribuídos como questões de insatisfação pelos usuários.

Gráfico 3 - Pontos de insatisfação em \% em cada área de conhecimento

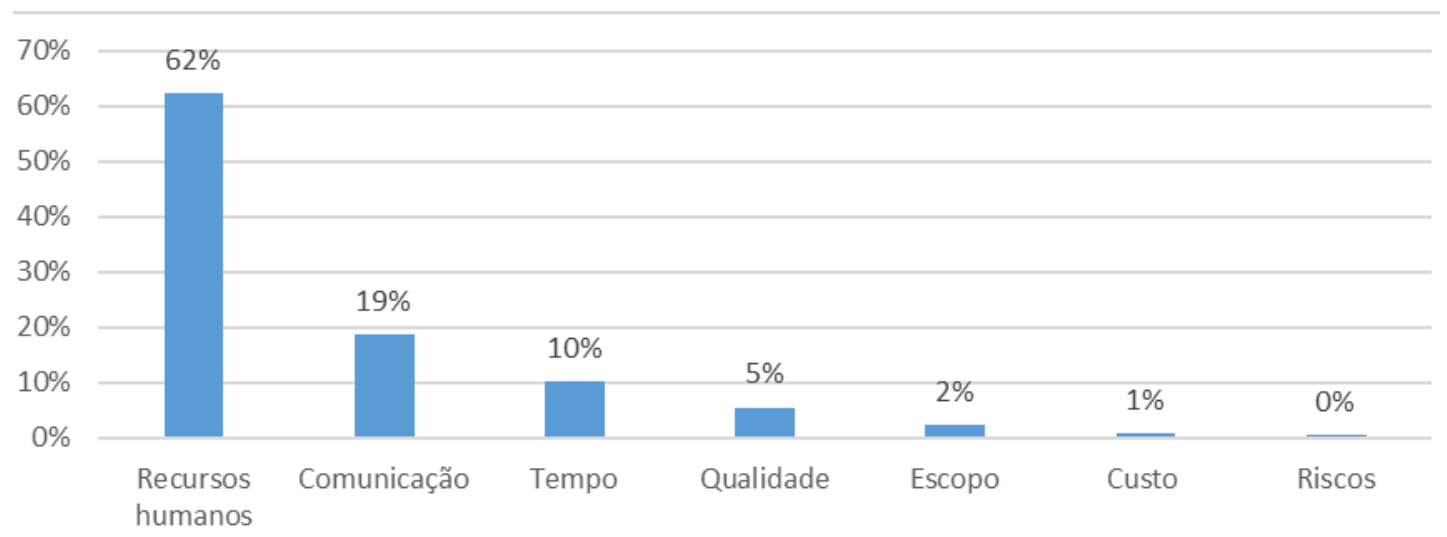

Fonte: Autor (2019) 


\section{Conclusão}

Uma variedade de funcionalidades são oferecidas por diferentes softwares de gerenciamento de projetos, as quais os usuários costumam avaliá-las em função de atender ao não às demandas dos seus projetos. Dessa forma, o objetivo deste trabalho não foi determinar uma ferramenta que melhor gerencie todos os projetos, mas sim identificar os pacotes de recursos disponibilizados em cada uma delas e verificar aqueles que são comuns a todos, aparecem apenas em algumas plataformas ou, ainda, não está disponível em nenhum dos softwares considerados pelo estudo.

Uma primeira crítica a ser realizada nesta área diz respeito ao grande número de plataformas que se intitulam como gerenciadores de projetos quando, na verdade, são ferramentas orientadas apenas para tarefas ou pessoas, ou seja, seus recursos e funções disponíveis são voltados para planejamento, monitoramento e controle de pessoas e atividades. O gerenciamento de atividades e pessoas são funções core da área de projetos, no entanto outras áreas de conhecimento em gerenciamento de projetos são tão importantes quanto, ajudando a garantir resultados mais assertivos e eficientes, tais como a gestão de risco, escopo - pouco exploradas pelas plataformas - e aquisição - sem registro entre as plataformas deste estudo.

Identificou-se uma oportunidade na área de aquisições, em razão da ausência de funções, entre as plataformas mais populares, que apoiassem a administração das aquisições e contratos de um projeto. Em grandes projetos, vários recursos precisam ser adquiridos a partir de contratos externos à organização, logo a falta de planejamento e controle destes poderão comprometer o sucesso do projeto. Dessa maneira, propõe-se que funcionalidades sejam pensadas nessa área de conhecimento, tornando as ferramentas mais poderosas para o gerenciamento dos projetos.

Notou-se que também existe uma grande oportunidade a ser explorada nas áreas de custos e riscos. Associado a isto, ferramentas de simulação integradas às plataformas também são alternativas de melhorias. Essas funcionalidades contribuiriam para a criação de cenários e tomada de decisões pelos gerentes de projetos.

Este trabalho trouxe contribuições para a área de sistema de informação em gestão de projetos, ao avaliar os atributos dos softwares mais utilizados no mercado atualmente pelos profissionais de projetos, trazendo à tona algumas oportunidades a fim de estreitar os hiatos nesta área. Vale acrescentar que essas ferramentas devam apoiar o processo de tomada de decisão, de forma que nem sempre será a decisão ótima, mas seja a melhor para aquele 
conjunto de variáveis considerada. Além disso, essas plataformas de gerenciamento de projetos dão visibilidade de todo o projeto para todos de forma flexível, ágil e inovadora, deixando de lado a forma tradicional de e-mails, planilhas e papel de gerenciar projeto.

Adicionalmente, verificamos que essas plataformas são intensamente utilizadas no setor da computação e informação para o gerenciamento do desenvolvimento de softwares e sistemas, realidade bem diferente foi constatada em relação aos setores tradicionais da indústria e serviços, nos quais o gerenciamento das informações e controle dos projetos se dar por meio de planilhas eletrônicas, manuais ou software populares como MS Project e Primavera. Frente a isso, vislumbra-se uma oportunidade para adequar as funcionalidades dessas plataformas às necessidades desses setores; assim, como futuros projetos, propõe-se entender as necessidades dos setores tradicionais - fábricas e serviços - e tornar as ferramentas que apoiam o gerenciamento de projetos mais próximas a realidade delas, considerando as suas particularidades.

\section{REFERÊNCIAS}

Best project management software 2019. Disponível em < https://technologyadvice.com/projectmanagement/\#best-project-management-software-2019>. Acesso em 01/09/2019.

BORGHOFF, U. W.; SCHLICHTER, J.H. Computer-Supported Cooperative Work: Introduction to Distributed Applications. Berlim: Springer. 2000.

BUDAYAN, C. ET AL. A Computerized Method for Delay Risk Assessment Based on Fuzzy set Theory using MS Project. KSCE Journal of Civil Engineering. v. 22(8). p. 2714-2725. 2018.

DUBININA, O. V.; HRYTSIAK, L. D. Use Of Information Technologies In Future Project Managers' Training. Information Technologies and Learning Tools. v. 63. p. 107-115. 2018.

GRAKHOV V.P., KISLYAKOVA Y.G., SIMAKOVA U.F., MUSHAKOV D.A. development of control system for progress of construction and installation works based on integrated application of primavera p6 professional r8.3.2 and archicad 17.0.0 software products. Science \& Technique. v.16(6).p. 466-474. 2017 (In Russ.) https://doi.org/10.21122/2227-1031-2017-16-6-466-474

ILYAS, M. A. B.; HASSAN, M. K.; ILYAS, M. U. PMIS: boon or bane. In: PMI® Global Congress EMEA, Istanbul, Turkey. Newtown Square, PA: Project Management Institute. (2013).

KACZOROWSKA, A.; SŁONIEC, J.; MOTYKA, S. IT solutions supporting project management processes and the choice of software. ITM Web of Conferences. 2017.

JUGEND, D.; BARBALHO, S. C. M.; DA SILVA, S. L. Gestão de Projetos: Teoria, Prática e Tendências. 1 ed. Rio de Janeiro - Elsevier, 2014

KHALIL, I. W.; SHAMAL, A. O. Comparison and Assessment of Using Primavera and Microsoft Project in Construction Projects in Erbil City. ZANCO Journal of Pure and Applied Sciences. v. 31. p. 285-291. 2019. 
Top 10 Best Project Management Software \& Tools in 2019. Disponível em <https://projectmanagement.com/top-10-project-management-software/> Acesso em 01/09/2019.

The 10 Best Project Management Tools Of 2019 Expert Review. Disponível em < https://thedigitalprojectmanager.com/best-project-management-software-tools/> Acesso em 01/09/2019.

The Best Project Management Software of 2019. Disponível em < https://www.pcmag.com/roundup/260751/thebest-project-management-software> Acesso em 01/09/2019

The 7 Best Project Management Software Tools of 2019. Disponível em <

https://www.thebalancesmb.com/best-project-management-software-4175032> Acesso em 01/09/2019

Project Management User Research Report. Disponível em < https://www.capterra.com/project-managementsoftware/user-research/> . Acesso em 01/08/2019.

PMI. GUIA PMBOK: Um Guia do Conjunto de conhecimentos em Gerenciamento de Projetos. 3 ed. 2004 REDDY, B. S. K.; NAGARAJU, S.K.; SALMAN, M.D. A Study On Optimisation Of Resources For Multiple Projects By Using Primavera. Journal of Engineering Science and Technology. v. 10(2). p. 235 - 248.2015

RUSHINEK, A.; RUSHINEK, S. A product evaluation and selection system for Project management software. Computers in Industry. v. 16. p.289-301. 1991.

SY, D. R. SharePoint for Project Management. United State of America: O’Reilly Media, 2009.

TAHERKHANI, R. Evaluating the Success of Project Management Information Systems' Comprehensive Planning in Industrialized Building Systems. Civil Engineering Journal-Tehran. v. 4. p. 1667-1677. 2018.

TURBAN, E; RAINER, R. R.; POTTER, R. E. Introduction to information technology. 3 ed. John Wiley \& Sons, 2005.

TURBAN, E.; DELEN, D.; SHARDA, R. Business Intelligence: A Managerial Perspective on Analytics. 3 ed. Pearson. 2014

Wellingtone PPM Inteligence. The state of Project Management Annual Survey 2018. Disponível em < http://www.wellingtone.co.uk/wp-content/uploads/2018/05/The-State-of-Project-Management-Survey-2018FINAL.pdf> Acesso em: 01/10/2019

WIT, J. D.; HERROELEN, W. An evaluation of microcomputer-based software packages for project management. European Journal of Operational Research. v. 49 (1). p.102-139. 1990.

ZHENG, L.; POSSEL-DÖLKEN, F. Strategic Production Networks. Belin: Springer, 2002.

50 Best Project Management Tools for 2019. Disponível em < https://www.proofhub.com/articles/top-projectmanagement-tools-list> Acesso em 01/09/2019.

42 Best Project Management Software and Tools. Disponível em < https://www.scoro.com/blog/best-projectmanagement-software-list> Acesso em 01/09/2019.

94 Essential Project Management Statistics: 2019 Analysis of Trends, Data and Market Share. Disponível em < https://financesonline.com/35-essential-project-management-statistics-analysis-of-trends-data-and-marketshare/> Acesso em 01/09/2019.

42 Best Project Management Software and Tools. Disponível em < https://www.scoro.com/blog/best-projectmanagement-software-list> Acesso em 01/09/2019.

17 Best Free Project Management Tools for You. Disponível em < https://www.fossmint.com/best-free-projectmanagement-tools/> Acesso em 01/09/2019.

40 BEST Project Management Tools. Disponível em < https://www.guru99.com/project-managementtools.html> Acesso em 01/09/2019. 